Article

\title{
A New Numerical Scheme with B-Spine Wavelet on the Interval for Transverse Vibration Problem of the Tethered Deep-Sea Robot
}

\author{
Naige Wang ${ }^{1}$ D and Xiaoqin Xiang ${ }^{2, *(D)}$ \\ 1 College of Mechanical and Electrical Engineering, Wenzhou University, Wenzhou 325035, China; \\ wangnaige@wzu.edu.cn \\ 2 Department of Humanities and Social Science, Wenzhou University, Wenzhou 325035, China \\ * Correspondence: xiangxiaoqin@wzu.edu.cn
}

check for updates

Citation: Wang, N.; Xiang, X. A New Numerical Scheme with B-Spine

Wavelet on the Interval for

Transverse Vibration Problem of the

Tethered Deep-Sea Robot. J. Mar. Sci. Eng. 2022, 10, 317. https://doi.org/ 10.3390/jmse10030317

Academic Editor: Alessandro Ridolfi

Received: 24 December 2021

Accepted: 13 February 2022

Published: 23 February 2022

Publisher's Note: MDPI stays neutral with regard to jurisdictional claims in published maps and institutional affiliations.

Copyright: (c) 2022 by the authors. Licensee MDPI, Basel, Switzerland. This article is an open access article distributed under the terms and conditions of the Creative Commons Attribution (CC BY) license (https:/ / creativecommons.org/licenses/by/ $4.0 /)$.

\begin{abstract}
This research is focused on the accurate modeling of a tethered deep-sea robot system with variable-length. Since the flexible umbilical cable is influenced by the surface vessel motion, the ocean current and sea states, etc., its transient response will reduce the deep-sea robot's stability. Thus, dynamic modeling of the elastic umbilical cable is a crucial issue. In this paper, transverse vibration of the tethered deep-sea robot system can be modelled as a one-dimensional distributed parameter system, a class of partial differential equations with nonhomogeneous boundary conditions theoretically. A new numerical scheme with B-spine wavelet on the interval (BSWI) is used to discretize and transform inhomogeneous partial differential equations into a set of ordinary differential equations and to obtain the dynamic response of the tethered deep-sea robot system with different ocean currents. Compared with conventional methods, BSWI finite element with multiresolution analysis principle can approximate the transverse vibration of the flexible umbilical cable better, and handle boundary conditions more easily. Numerical examples of different cases are analyzed in detail by the discussion of an ADAMS model, and simulation results of the ADAMS model also verify that BSWI finite element method has a desirable performance than other methods.
\end{abstract}

Keywords: deep-sea robot; transverse vibration; BSWI finite element; nonhomogeneous partial differential equations

\section{Introduction}

A tethered deep-sea robot system, usually composed of a surface vessel, an umbilical cable, and an underwater robot, is used to provide submarine exploration, seabed mineral collection, and offshore oil development, which is ascended and descended by the flexible umbilical cable as Figure 1 [1]. However, it is faced with complex environmental factors like sea waves and ocean current, etc., shortening the service life of the umbilical cable since it causes excessive transverse vibration during the ascending or descending process [2].

When bending stiffness is neglected, the transverse vibration problem of the umbilical cable with axial velocity can be modeled as a one-dimensional continuum vibration string [3]. Variable-length and axial velocity are two main factors causing structural vibration [4], since the former will produce a change of tension in the axial direction and the latter will introduce translational and rotational distribution gyro force called as Coriolis Effects acting on moving bodies [5,6]. Therefore, the transverse vibration of the thered deep-sea robot system calculation for the understanding of flexible vibration mechanism to prevent the excessive vibration and ensure the safety and stability of the system. When the ocean current generally flows in one direction, the result of the umbilical cable is different from that produced by periodic disturbance, where the flexible umbilical cable could have spatial shape deformation under the ocean current, and it will cause the phenomenon of severe alternating stress under the high sea state $[3,7]$. So far, the reliability model and analysis 
method of the continuum vibration are well documented and published in the literature [8]. For transverse vibration problem of moving strings with fixed length, a series of expansions were used to obtain the solution of moving strings under some simple boundary conditions by Mote [9]. The author has done further study on nonlinear vibration of the axially moving medium and established the government equations by using energy-based method, such as Hamilton principle [10]. Energy-based method is a more comprehensive evaluation tool compared with material mechanics method for structural vibration problem [11].

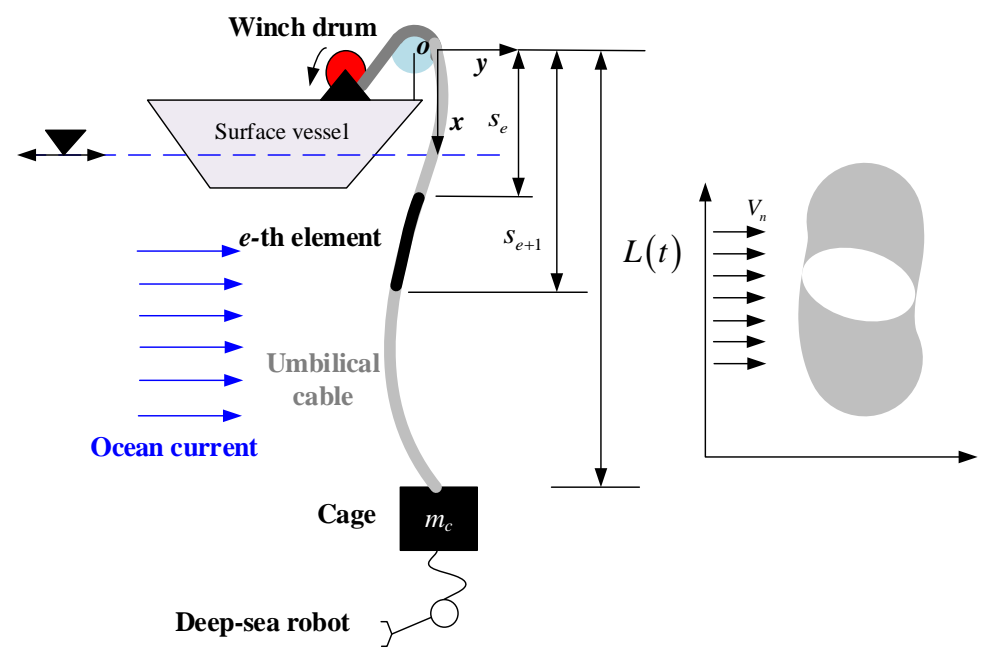

Figure 1. Deep-sea robot and hydrodynamic disturbance.

There are four basic computation methods proposed to solve partial differential equations (PDEs), including Galerkin method, finite difference method (FDM), least square method, and finite element method (FEM), etc. [12,13], seeking approximate solutions with a linear combination of appropriate approximation functions satisfies the weak form or the second functional equation minimized [14]. Galerkin method and finite element method stand out of high calculation accuracy and development efficiency. However, the Galerkin method has a deficiency that the basic function needs to satisfy the boundary conditions. Compared with Galerkin method, FEM has the advantage that the element function does not need to satisfy the boundary conditions. Based on the system characteristics, a onedimensional element can be generally divided into $C^{0}$ or $C^{1}$ type elements [15], where the $C^{0}$ type element requires the least zero-order continuously differential interpolation function (i.e., Lagrange type), the $C^{1}$ element known as Hermite type requires the least one order continuously differential interpolation function. To reduce computation cost, the appropriate type element should be selected according to an order of partial derivative of the model [16].

Many current research works have been carried out on numerical simulation of the kinematic and dynamic characteristics of the umbilical cable in the tethered deep-sea robot system of the process of underwater operation. Still, very few have studied on the system ascending or descending process $[17,18]$. Therefore, a further understanding on mechanical vibration with variable parameters is critical to the optimal control design [19]. A series of interpolation functions can be selected as the wavelet-based function, where Daubechies and Hermite wavelets are the two most famous interpolation wavelet-based functions. The former lacks explicit expression and sufficient regularity, so it's hard to normalize the program. Although the Hermite wavelet has explicit terms, it is inferior to BSWI finite element on numerical approximation [20].

Compared with the Galerkin method, BSWI finite element method converts the wavelet interpolation coefficient, without definite physical meaning, into the physical freedom of the finite element space through the dimensional transformation matrix. The non-singularity transformation matrix guarantees the stability of the solution [21]. Displacement of the deep-sea robot is time-varying with the cable length, and its space-time 
coupled finite element formulations are not common and have not been adequately studied. There are two approaches to describe the time-varying space domain, one is an Eulerian-Lagrangian approach, and the other is an Eulerian approach [22]. Compared with traditional methods, trial function and test function are replaced by the boundary scaling functions for BSWI the nodal variable, where it was proved in practice that the BSWI function is better than the other interpolation functions in converting nodal displacement into wavelet coefficients by continuous wavelet transform and in multi-resolution processing of weak signals by different wavelet scaling function. There are three main differences between BSWI finite element method and other existing traditional methods: first, it solves the equation directly without converting the non-homogeneous equation into homogeneous partial differential equation; second, the wavelet coefficients can be mapped into physical space by the constructed transformation matrix; finally, the mixed Dirichlet-Neumann boundary which failed solved by existing FEM can be handled by the transformation matrix in the present FEM $[23,24]$.

The paper is arranged as following: Section 2 provides the basic theory of BWSI $[0,1]$. The hydrodynamic disturbance is the key factor causing spatiotemporal vibration for the tethered deep-sea robot in Section 3. Transverse vibration modelling of the system is derived with variable-length BSWI finite element format in Section 4, followed by the verified by the ADAMS simulation in Section 5. In Section 6, some typical different cases of the hydrodynamic disturbance are given to verify the advantages of the proposed method. Several conclusions are given in last section.

\section{BSWI Finite Element}

The multiresolution analysis and wavelets on a bounded interval were developed by Chui et al. $[25,26]$, a recursive formula of $m$-order B-spine wavelet is defined as

$$
N_{m}(x)=\frac{x}{m-1} N_{m-1}(x)+\frac{m-x}{m-1} N_{m-1}(x-1)
$$

where, $N_{m}(x)$ is a compactly supported function, when $N_{1}$ (i.e., $m=1$ ) is the initial value function, equals to one only in the semi-open interval $[0,1)$ and the remaining interval is equal to zero.

The scaling function consists of interval spline functions, where the spline function formed by a set of node sequence $x j$ forms B spline function space with $\mathrm{m}$ order, and its basic function is expressed as

$$
B_{m, k}^{j}(\xi):=\left(x_{k+m}^{j}-x_{k}^{j}\right) \times\left[x_{k^{\prime}}^{j} x_{k+1}^{j}, \cdots, x_{k+m}^{j}\right]_{x}(x-\xi)_{+}^{m-1}
$$

where, the compactly supported set $B_{m, k}^{j}(\xi)$ can be expressed as $\operatorname{supp} B_{m, k}^{j}(\xi)=\left[x_{k}^{j}, x_{k+m}^{j}\right]$, and $\left[x_{k}^{j}, x_{k+1}^{j}, \cdots, x_{k+m}^{j}\right]_{x}$ is $m$ order difference of the variable $(x-\xi)_{+}^{m-1}$, in which $(x-\xi)_{+}=\max \{0, x-\xi\}$.

Then, the space of the $m$ order $j$ scaling BSWI is given as

$$
S_{m, x j}^{j}:=\left\{s \in C^{m-2}[0,1]: s_{\mid\left(x_{k}^{j}, j_{k+1}^{j}\right)} \in \Pi^{m-1}\left(k=0,1, \cdots, 2^{j}-1\right)\right\}
$$

where, $\Pi^{m-1}$ stands for the polynomial space of $m-1$ order interpolation function.

The boundary scaling function $\phi(\xi)$ is the B-spline truncated function on the interval $[0,1]$. For each order $m$ of BSWI, scale $j$ of the scaling function $\phi_{m, k}^{j}(\xi)$ need to be selected as the following inequality to guarantee that at least one inner wavelet as

$$
2^{j} \geq 2 m-1
$$


The $j$ scale BSWI scaling function can be transformated by zero scale at $m$-th order BSWI scaling functions (simplified as BSWI $m_{j}$ ) given as:

$$
\phi_{m, k}^{j}(\xi)= \begin{cases}\phi_{m, k}^{l}\left(2^{j-l} \xi\right), & k=-m+1, \cdots,-1(0 \text { boundary functions }) \\ \phi_{m, 0}^{l}\left(2^{j-l} \xi-2^{-l} k\right), & k=0, \cdots, 2^{j}-m(\text { inner boundary functions }) \\ \phi_{m, 2^{j}-m-k}^{l}\left(1-2^{j-l} \xi\right), & \left.k=2^{j}-m+1, \cdots, 2^{j}-1 \text { (1 boundary functions }\right)\end{cases}
$$

where, $j_{0}$ is the smallest integer that satisfies Equation (4).

The compact supports of BWSI are given as

$$
\operatorname{supp} \phi_{m, k}^{j}(\xi)=\left\{\begin{array}{cc}
{\left[0,(2 m-1+k) 2^{-j}\right]} & (0 \text { boundary wavelets }) \\
{\left[k 2^{-j},(2 m-1+k) 2^{-j}\right]} & (\text { inner wavelets }) \\
{\left[k 2^{-j}, 1\right]} & (1 \text { boundary wavelets })
\end{array}\right.
$$

Such that $l=0$, the scaling functions can be derived by Equation (5) for each $j>j_{0}$. There are $m-1$ boundary scaling functions at both ends of the boundary $(\xi=0$ or $\xi=1)$, respectively, and $2^{j}-m+1$ inner scaling function on the internal domain. BSWI $m_{j}$ represents the interpolation function with the order $m$ and scaling function $j$, where two typical BSWI functions with different orders are depicted in Figure 2. Since even-order interpolation function has orthogonal symmetry with compact support proposed in the numerical simulation, we try to choose low order wavelet interpolation function to meet precision requirement.
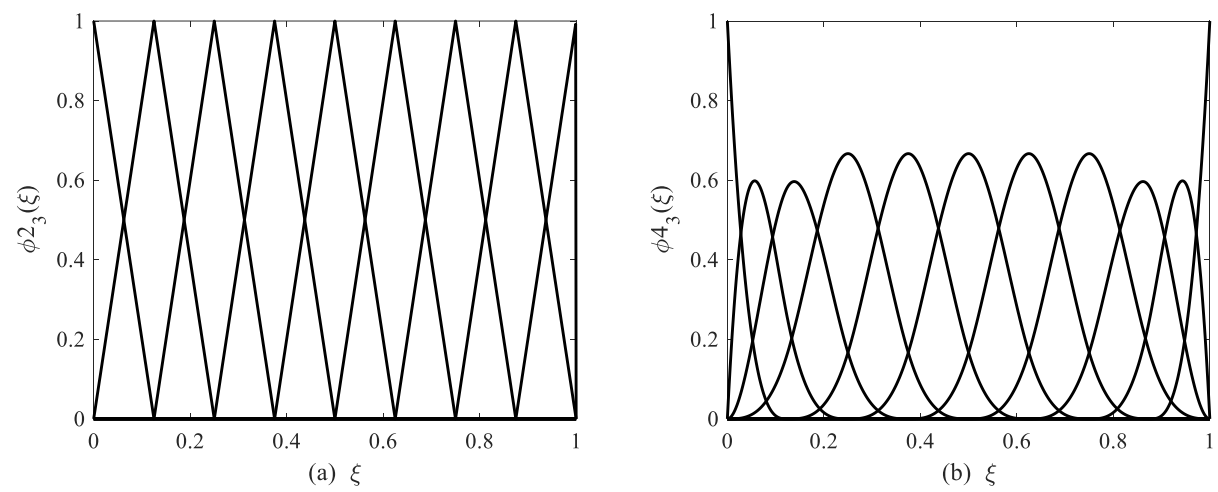

Figure 2. BSWI scaling functions using different orders on the interval $[0,1](\mathbf{a})$ BSWI23 (b) BSWI43 [15,16].

\section{Hydrodynamic Disturbance}

Umbilical cable interacting with ocean flows, the dynamic force from the ocean waves and currents is a hydrodynamic problem in small diameter rod. When the diameter of the umbilical cable is smaller than the wavelength, the current velocity $V_{n}$ in a steady motion is dependent with water depth $l(t)$ as

$$
V_{n}=V_{n 0}+V_{n 1}\left(1-l(t) / L_{0}\right)^{12}
$$

where, $V_{n 0}$ and $V_{n 1}$ are ocean current parameters. The relation Equation (7) implies that the current velocity diminish with the sea depth increases.

Since the material particles of the seawater move in the direction of the velocity field, they cannot cross the boundary of the umbilical cable, which will act on the entire cable called as drag force. They can be decomposed into normal and tangential force. Due to tangential force being smaller than the normal component, and only normal disturbance force is considered in this paper. When the cross section of the umbilical cable is circular 
and symmetrical, the Morison equation is recommended to calculate distributed drag force $f$ with circular cross section. These forces are defined as follows:

$$
f=\frac{1}{2} C_{D} \rho_{w} D\left|V_{n}\right| V_{n}
$$

where, $C_{D}$ and $\rho_{w}$ are normal drag coefficient and seawater density, respectively. $D$ is the diameter of the umbilical cable. A lot of systematic experiments were done to study the drag coefficient of underwater cable interacting with ocean flows by Sarpkaya [27], where the experiment results show that the normal drag coefficient $C_{D}$ varies within a specific interval in $[0.6,1.2]$.

\section{The Finite Element Model of the Deep-Sea Robot for BSWI Finite Element}

The transverse vibration problem of the tethered deep-sea robots with variable-length in motion. It is assumed that the BSWI finite element coefficient to be solved in the equation is a time-dependent function, and the separation variables depend on the spatial coordinates. Transverse vibration is firstly considered into the space approximation, then considered into the time approximation, such a process is generally called semi-discrete approximation. The basic idea is to use the BSWI finite element to transform the physical space into the wavelet space through the transformation matrix. A set of time-dependent coupled differential equations is obtained in the semi-discrete space, which can be solved by numerical calculation.

\subsection{BSWI Finite Element Nodal Displacement}

The transverse vibration of the umbilical cable is a wave propagation dynamics problem with varying length, and it can be approximated by using BSWI boundary element method. The strategy of solving this problem is to discretize the moving umbilical cable as multiple elements, and the length of each element increases or decreases with time. Hence, the axial moving cable elements are constructed by utilizing Liebnitz's rule. Finite element models of the time-dependent problems with time-varying coefficient can be developed in coupled formulation, where the time $t$ is treated as an additional coordinate along the spatial coordinate $x$, the scaling function $\boldsymbol{\Phi}(\xi, t)$ is time-space interpolation functions, and the wavelet coefficients $q^{e}(t)$ are element nodal values that is independent of time $t$.

Using BSWI finite element to discrete space, the space domain of the umbilical cable should be divided into a set of subintervals. The subintervals of $i$-th element are located between nodal global coordinate $s_{e}(t)$ and $s_{e+1}(t)$, (i.e., the element length $\left.l_{e}(t)=s_{e+1}(t)-s_{e}(t)\right)$ donated as

$$
x=\left[s_{e}(t), s_{e+1}(t)\right], 1 \leq e \leq n+1
$$

First, define $\xi=\left(x-s_{e}(t)\right) / l_{e}(t)$, then time variant domain $\left[s_{e}(t), s_{e+1}(t)\right]$ can be transformed to $\xi \in[0,1]$. Any point in space $x$ represented by $\xi$ is given as

$$
x=X(\xi, t)=s_{e}(t)+\xi\left[s_{e+1}(t)-s_{e}(t)\right]=s_{e}(t)+\xi l_{e}(t), x \in\left[s_{e}, s_{e+1}\right]
$$

The transverse displacement of the umbilical cable $Y(x, t)$ becomes $y(\xi, t)=Y(X(\xi, t)$, $t)=Y(x, t)$. Its first derivative and higher-order partial derivative respect with $t$ and $x$

$$
\begin{gathered}
\frac{\partial Y(x, t)}{\partial t}=\frac{\partial y(\xi, t)}{\partial t}-\frac{\dot{s}_{e}+v_{e} \xi}{l_{e}(t)} \frac{\partial y(\xi, t)}{\partial \xi}, \frac{\partial Y(x, t)}{\partial x}=\frac{1}{l_{e}(t)} \frac{\partial y(\xi, t)}{\partial \xi} \\
\frac{\partial^{k+l} Y(x, t)}{\partial x^{k} \partial t^{\mu}}=\frac{1}{l_{e}(t)^{k}} \frac{\partial^{k}}{\partial \xi^{k}}\left(\frac{\partial}{\partial t}-\frac{\dot{s}_{e}+v_{e} \xi}{l_{e}(t)} \frac{\partial}{\partial \xi}\right)^{\mu} y(\xi, t)
\end{gathered}
$$

where, an overdot denotes a time derivative, $k$ and $\mu$ are two non-negative integers. 
To satisfy the compatibility and continuity of the displacement of adjacent boundary elements, the transverse vibration $y(\xi, t)$ of the umbilical cable function is an unknown field, and it can be obtained by substituting the approximation as

$$
y(\xi, t)=N^{e}(\xi, t) q^{e}(t)=\boldsymbol{\Phi}(\xi, t) \boldsymbol{T}^{e}(\xi) \boldsymbol{q}^{e}(t)
$$

where, $\boldsymbol{\Phi}(\xi, t) \in \mathbb{R}^{1 \times \beta}$ is a row vector of the wavelet basis function; $\boldsymbol{T}^{e}(\xi) \in \mathbb{R}^{\alpha \times \beta}$ is a transformation matrix of $C^{1}$ type element as

$$
\boldsymbol{T}^{e}(\xi)=\left[\begin{array}{llll}
\boldsymbol{\Phi}^{\mathrm{T}}\left(\xi_{1}\right) & \boldsymbol{\Phi}^{\mathrm{T}}\left(\xi_{2}\right) & \cdots & \boldsymbol{\Phi}^{\mathrm{T}}\left(\xi_{n+1}\right)
\end{array}\right]^{-1}
$$

Noting that the transformation matrix $T(\xi)$ is a nonsingular constant matrix, which transforms the wavelet space to physical space and handles two boundary conditions conveniently. The following section gives a more detail by using BSWI finite element to build a model with time-varying domain for the tethered deep-sea robot system.

\subsection{Government Equation of the System}

The finite element method is applied to the variable time-domain string element, and an inertial coordinate axis is established at the upper end of the umbilical cable, whose transverse displacement is known in the spatial description, $Y(x, t)$. Its time derivative is known as the material derivative [28]. Definition $\epsilon=(\partial Y / \partial x)^{2} / 2$ represents the nonlinearity Green's symmetric strain tensor for the umbilical cable. For the $s_{e}(t)$ and $s_{e+1}(t)$ are two boundaries of a string finite element, which is also a function of time. Coriolis force will generate the mixed partial derivatives of space domain and time. The kinetic energy with linearly distributed axial velocity and potential energy of the axial variable length of the umbilical cable in each element can be expressed as $K_{p}$ and $U_{p}$, respectively [29].

$$
\begin{gathered}
K_{p}=\sum_{e=1}^{n} \frac{\rho}{2} \int_{s_{e}}^{s_{e+1}}\left(\frac{\partial Y}{\partial t}+\varepsilon v \frac{\partial Y}{\partial x}\right)^{\mathrm{T}}\left(\frac{\partial Y}{\partial t}+\varepsilon v \frac{\partial Y}{\partial x}\right) \mathrm{d} x+\frac{1}{2} m_{c} Y_{t}(l, t)^{2} \\
U_{p}=\sum_{e=1}^{n} \int_{s_{e}}^{s_{e+1}} P(x, t) \epsilon \mathrm{d} x \\
\delta W_{p}=f \delta Y
\end{gathered}
$$

where, notation $\varepsilon$ is a small constant parameter for each element, which is less than or equal to one. $P(x, t)$ stands for the axial force in the umbilical cable, $P(x, t)=\left[m_{c}+\rho(L(t)-x)\right](g-a)$, which is linearly distributed as the umbilical cable length increases during descend process.

Applying the extended Hamiltonian principle, the system dynamic model is a timedependent hyperbolic PDEs (i.e., second-time derivative), where the government equation of the system can be written as [19]

$$
\begin{gathered}
\rho \frac{D^{2} Y(x, t)}{D t^{2}}-\frac{\partial}{\partial x}\left[T(x, t) \frac{\partial Y(x, t)}{\partial x}\right]=f, s_{e}(t)<x<s_{e+1}(t) \\
Y(x, t)=e(t), x=0 \\
m_{c} \frac{D^{2} Y(x, t)}{D t^{2}}+P(x, t) \frac{\partial Y(x, t)}{\partial x}=0, x=L(t)
\end{gathered}
$$

where, $\frac{D^{2} Y(x, t)}{D t^{2}}=Y_{t t}(x, t)+2 \varepsilon v Y_{x t}(x, t)+\varepsilon^{2} v^{2} Y_{x x}(x, t)+\varepsilon a Y_{x}(x, t)$.

For a long variable-length cable consisting of $n$ cable elements, there are mass flows at both ends. When a uniform mesh is used to the whole umbilical cable at an arbitrary time $t$, the length of every element is $l_{e}(t)=L(t) / n$. Then, we get that

$$
\frac{\partial Y(x, t)}{\partial t}=y_{t}(\xi, t)-\frac{\dot{s}_{e}+v_{e} \xi}{l_{e}} y_{\xi}(\xi, t), \frac{\partial Y(x, t)}{\partial x}=\frac{1}{l_{e}} y_{\xi}(\xi, t)
$$




$$
y_{t}=\boldsymbol{\Phi} \boldsymbol{T}^{e} \dot{\boldsymbol{y}}^{e}, y_{t t}=\boldsymbol{\Phi} \boldsymbol{T}^{e} \ddot{\boldsymbol{y}}{ }^{e}, y_{\xi}=\boldsymbol{\Phi}_{\xi} \boldsymbol{T}^{e} \boldsymbol{y}^{e}, y_{\xi \xi}=\boldsymbol{\Phi}_{\xi \xi} \boldsymbol{T}^{e} \boldsymbol{y}^{e}, y_{\xi t}=\boldsymbol{\Phi}_{\xi} \boldsymbol{T}^{e} \dot{\boldsymbol{y}}^{e}
$$

Substitution Equations (6) and (13) into Equations (15)-(17), respectively, we obtain

$$
\begin{gathered}
K_{p}=\sum_{e=1}^{n}\left(\frac{1}{2} \dot{\boldsymbol{q}}^{e \mathrm{~T}} \boldsymbol{M}^{e} \dot{\boldsymbol{q}}^{e}-\dot{\boldsymbol{q}}^{e \mathrm{~T}} \boldsymbol{G}^{e} \boldsymbol{q}^{e}+\frac{1}{2} \boldsymbol{q}^{e \mathrm{~T}} \boldsymbol{K}^{e} \boldsymbol{q}^{e}\right)+\frac{1}{2} \dot{\boldsymbol{q}}_{c}^{e \mathrm{~T}} \boldsymbol{M}_{c}^{e} \dot{\boldsymbol{q}}_{c}^{e} \\
U_{p}=\sum_{e=1}^{n} \frac{1}{2} \boldsymbol{q}^{e \mathrm{~T}} \boldsymbol{K}_{2}^{e} \boldsymbol{q}^{e}
\end{gathered}
$$

where the transverse stiffness submatrix of the $e$-th element $\boldsymbol{K}_{j}^{e}, j=1,2,3$ are given as

$$
\boldsymbol{K}_{1}^{e}=\rho \frac{1}{l_{e}} \boldsymbol{T}^{e \mathrm{~T}} \int_{0}^{1}\left(\varepsilon v-\left(\dot{s}_{e}+v_{e} \xi\right)\right)^{2} \boldsymbol{\Phi}_{\xi}^{\mathrm{T}} \boldsymbol{\Phi}_{\xi} \mathrm{d} \xi \boldsymbol{T}^{e}, \boldsymbol{K}_{2}^{e}=\frac{1}{l_{e}} \boldsymbol{T}^{\mathrm{T}} \int_{0}^{1} P^{e}(\xi, t) \boldsymbol{\Phi}_{\xi}^{\mathrm{T}} \boldsymbol{\Phi}_{\xi} \mathrm{d} \xi \boldsymbol{T}^{e},
$$

The element mass submatrix $\boldsymbol{M}^{e}$ and lumped mass submatrix of the deep robot $\boldsymbol{M}_{\mathcal{C}}^{e}$ are

$$
\boldsymbol{M}^{e}=\rho l_{e} \boldsymbol{T}^{e \mathrm{~T}} \int_{0}^{1} \boldsymbol{\Phi}^{\mathrm{T}} \boldsymbol{\Phi} \mathrm{d} \xi \boldsymbol{T}^{e}, \boldsymbol{M}_{c}^{e}=m_{c} \boldsymbol{T}^{e \mathrm{~T}} \boldsymbol{\Phi}(1)^{\mathrm{T}} \boldsymbol{\Phi}(1) \boldsymbol{T}^{e}
$$

and the element gyroscopic submatrix $G^{e}$ derived from axial velocity is

$$
\boldsymbol{G}^{e}=\rho \boldsymbol{T}^{e \mathrm{~T}} \int_{0}^{1}\left[\left(\dot{s}_{e}+v_{e} \xi\right)-\varepsilon V\right] \boldsymbol{\Phi}_{\xi}^{\mathrm{T}} \boldsymbol{\Phi}_{\xi} \mathrm{d} \xi \boldsymbol{T}^{e}
$$

Upon applying Lagrange's equation to the element Lagrangian function $L_{p}=K_{p}-U_{p}$, Equations (18)-(20) can be discretized into the multivariant differential equation groups. Then, the total nodal force vector and total mass submatrices and stiffness submatrices by assembling every finite element, and the transient vibration finite element equations of the umbilical cable are derived as

$$
\overline{\boldsymbol{M}} \frac{\partial^{2} \boldsymbol{y}}{\partial t^{2}}+\overline{\boldsymbol{C}} \frac{\partial y}{\partial t}+\overline{\boldsymbol{K}} \boldsymbol{y}=\boldsymbol{F}
$$

In which,

$$
\overline{\boldsymbol{M}}=\left[\begin{array}{llll}
\boldsymbol{M}^{1} & & & \\
& \ddots & & \\
& & \boldsymbol{M}^{n-1} & \\
& & & \boldsymbol{M}^{n}+\boldsymbol{M}_{c}^{n}
\end{array}\right], \overline{\boldsymbol{C}}=\left[\begin{array}{cccc}
\boldsymbol{C}^{1} & & & \\
& \ddots & & \\
& & \boldsymbol{C}^{n-1} & \\
& & & \boldsymbol{C}^{n}
\end{array}\right], \overline{\boldsymbol{K}}=\left[\begin{array}{lll}
\boldsymbol{K}^{1} & & \\
& \ddots & \\
& & \boldsymbol{K}^{n-1} \\
& & \boldsymbol{K}^{n}
\end{array}\right]
$$

where, the element stiffness matrix $\boldsymbol{K}^{e}$, damping matrix $C^{e}$ and mass matrix $\boldsymbol{M}^{e}$ are defined as

$$
\begin{aligned}
\boldsymbol{M}^{e} & =\rho l_{e} \boldsymbol{A}_{e}, \quad \boldsymbol{M}_{c}^{n}=m_{c} \boldsymbol{A}_{e}(1), \quad \boldsymbol{f}^{e}=l_{e} f \boldsymbol{J}_{e} \\
\boldsymbol{C}^{e} & =\rho \varepsilon V\left(\boldsymbol{B}_{e}-\boldsymbol{B}_{e}^{\mathrm{T}}\right)-2 \rho\left(\dot{\mathrm{s}}_{e} \boldsymbol{B}_{e}+v_{e} \boldsymbol{H}_{e}\right)+\rho v_{e} \boldsymbol{A}_{e} \\
\boldsymbol{K}^{e} & =\rho \frac{\dot{s}_{e}^{2} \boldsymbol{G}_{e}+2 \dot{s}_{e} v_{e} \boldsymbol{H}_{e}+v_{e}^{2} \boldsymbol{K}_{e}}{l_{e}}-\rho \varepsilon V \frac{\dot{s}_{e} \boldsymbol{G}_{e}+v_{e} \boldsymbol{H}_{e}}{l_{e}}+\rho \varepsilon\left(a-V \frac{v_{e}}{l_{e}}\right) \boldsymbol{B}_{e} \\
& -\rho\left(\left(\ddot{s}_{e} \boldsymbol{B}_{e}+a_{e} \boldsymbol{H}_{e}\right)-2 \frac{v_{e}}{l_{e}}\left(\dot{s}_{e} \boldsymbol{B}_{e}+v_{e} \boldsymbol{H}_{e}\right)\right)+\left(-\rho v_{e} \frac{\dot{s}_{e} \boldsymbol{B}_{e}+v_{e} \boldsymbol{H}_{e}}{l_{e}}+\rho \varepsilon v_{e} V \frac{1}{l_{e}} \boldsymbol{B}_{e}\right) \\
& +\rho \varepsilon V \frac{\dot{s}_{e} \boldsymbol{D}_{e}+v_{e} \boldsymbol{E}_{e}}{l_{e}}-\rho(\varepsilon V)^{2} \frac{1}{l_{e}} \boldsymbol{D}_{e}+\frac{m+\rho l_{e}(n-e)}{l_{e}}(g-a) \boldsymbol{D}_{e}+\rho(g-a) \boldsymbol{F}_{e}
\end{aligned}
$$

where, the internal integral terms are given as

$$
\begin{aligned}
& \boldsymbol{A}_{\boldsymbol{e}}=\boldsymbol{T}^{e \mathrm{~T}} \int_{0}^{1} \boldsymbol{\Phi}^{\mathrm{T}} \boldsymbol{\Phi} \mathrm{d} \xi \boldsymbol{T}^{e}, \boldsymbol{B}_{e}=\boldsymbol{T}^{e \mathrm{~T}} \int_{0}^{1} \boldsymbol{\Phi}^{\mathrm{T}} \frac{\mathrm{d} \boldsymbol{\Phi}}{\mathrm{d} \xi} \mathrm{d} \xi \boldsymbol{T}^{e}, \boldsymbol{H}_{e}=\boldsymbol{T}^{e \mathrm{~T}} \int_{0}^{1} \xi \boldsymbol{\Phi}^{\mathrm{T}} \frac{\mathrm{d} \boldsymbol{\Phi}}{\mathrm{d} \xi} \mathrm{d} \xi \boldsymbol{T}^{e} \\
& \boldsymbol{D}_{e}=\boldsymbol{T}^{e \mathrm{~T}} \int_{0}^{1}\left(\frac{\mathrm{d} \boldsymbol{\Phi}}{\mathrm{d} \xi}\right)^{\mathrm{T}} \frac{\mathrm{d} \boldsymbol{\Phi}}{\mathrm{d} \xi} \mathrm{d} \xi \boldsymbol{T}^{e}, \boldsymbol{E}_{e}=\boldsymbol{T}^{e \mathrm{~T}} \int_{0}^{1} \xi\left(\frac{\mathrm{d} \boldsymbol{\Phi}}{\mathrm{d} \xi}\right)^{\mathrm{T}} \frac{\mathrm{d} \boldsymbol{\Phi}}{\mathrm{d} \zeta} \mathrm{d} \xi \boldsymbol{T}^{e}, \\
& \boldsymbol{F}_{e}=\boldsymbol{T}^{e \mathrm{~T}} \int_{0}^{1}(1-\xi) \boldsymbol{T}^{e \mathrm{~T}}\left(\frac{\mathrm{d} \boldsymbol{\Phi}}{\mathrm{d} \xi}\right)^{\mathrm{T}} \frac{\mathrm{d} \boldsymbol{\Phi}}{\mathrm{d} \xi} \boldsymbol{T}^{e} \mathrm{~d} \xi \boldsymbol{T}^{e}, \boldsymbol{G}_{e}=\boldsymbol{T}^{e \mathrm{~T}} \int_{0}^{1} \boldsymbol{\Phi}^{\mathrm{T}} \frac{\mathrm{d}^{2} \boldsymbol{\Phi}}{\mathrm{d} \xi^{2}} \mathrm{~d} \xi \boldsymbol{T}^{e}, \boldsymbol{J}_{e}=\int_{0}^{1} \boldsymbol{T}^{e \mathrm{~T}} \boldsymbol{\Phi}^{\mathrm{T}} d \xi
\end{aligned}
$$




\subsection{Natural Frequency}

For natural vibration, the natural frequency of the system is related to the system mass, damping and stiffness, and is independent of external disturbance [30,31]. Assuming that the deep-sea robot system is linear, we define a system state vector $X$ and state equation $\operatorname{matrix} A$ as $\boldsymbol{X}=[\dot{\boldsymbol{y}}, \boldsymbol{y}]^{\mathrm{T}}, \boldsymbol{A}=\left[\begin{array}{cc}-\overline{\boldsymbol{M}}^{-1} \overline{\boldsymbol{C}} & -\overline{\boldsymbol{M}}^{-1} \overline{\boldsymbol{K}} \\ I & 0\end{array}\right]$.

Transform the system equation with into first-order state equation $\dot{X}=A X$, its eigenvalue problem is given by

$$
|\boldsymbol{A}-\lambda \boldsymbol{I}|=\left|\begin{array}{cc}
-\overline{\boldsymbol{M}}^{-1} \overline{\boldsymbol{C}}-\lambda \boldsymbol{I} & -\overline{\boldsymbol{M}}^{-1} \overline{\boldsymbol{K}} \\
\boldsymbol{I} & -\lambda \boldsymbol{I}
\end{array}\right|=\left|\overline{\boldsymbol{M}}^{-1}\right| \cdot\left|\lambda^{2} \overline{\boldsymbol{M}}^{-1}+\lambda \overline{\boldsymbol{C}}+\overline{\boldsymbol{K}}\right|
$$

where, $\lambda$ is the eigenvalue of the system. Since $\overline{\boldsymbol{M}}$ is a positive definite matrix, then $\left|\overline{\boldsymbol{M}}^{-1}\right| \neq 0$. It may have complex eigenvalues when damping $\overline{\boldsymbol{C}}$ is included.

\section{Model Verification}

A fixed space coordinate system $O-x y$ is established at the initial position of the surface vessel for convenience. A winch machine, placed mounted on the upper deck of the surface vessel control the length of the umbilical cable. There has some distance between wound point and the sea surface. Thus, initial length of the underwater umbilical cable is set as $10 \mathrm{~m}$ to estimate the dynamic response of the tethered deep-sea robot system in seawater, where the cable diameter is $d=20 \mathrm{~mm}$, material density is $\rho_{s}=7800 \mathrm{~kg} / \mathrm{m}^{3}$, the mass of the deep-sea robot is $500 \mathrm{~kg}$. It is forced vibration with external boundary disturbance and distributed disturbance. The mass of the surface vessel is considered infinite.

Quasi-static and boundary disturbance of the four-level oceanic condition are illustrated to verify transverse vibration of the umbilical cable with time-variable length, where the quasi-static condition means that the surface vessel does not basically drift, and boundary disturbance of the four-level oceanic condition represents that the surface vessel sails in rough sea condition. The definite integral calculation of Equation (30) consists of the wavelet scaling function, whose expressions are exact and piecewise functions from 0 to 1 shown as Figure 2. As the general process of the definite integral calculation is exceptionally complicated by using Newton Leibniz Law, which is replaced by Gauss-Legendre numerical method with multiple integral points. Above differential equations are solved to obtain the dynamic vibration of the tethered deep-sea robot by using explicit Runge-Kutta formulas of orders 4 and 5 (RK45) with zero initial states and the time step is $\Delta t=10^{-3} \mathrm{~s}$.

The frequency response characteristics are closely related to the dynamic performance of the umbilical cable. For damped systems, the roots of the eigenvalue problem are complex number, where the real part represents the decay rate of the system and the imaginary part represents the natural frequency of the system. The expression results of normalized umbilical length as horizontal axis and natural frequency as vertical coordinates are shown in Figure 3. It can be seen from the figure that the transverse frequency of the system decreases gradually with the variable length of the umbilical cable.

While the surface vessel sailing in rough sea condition, slamming will happen between the hull and waves, which could reduce and break the system stability of the umbilical cable in severe cases. According to the sea state parameters of the four-level oceanic condition, the period and height of the sea wave are given as $10 \mathrm{~s}$ and $0.785 \mathrm{~m}$, respectively.

Two methods are used to in spatial and time discretization: the first approach uses the BSWI boundary finite element, and the second approach uses traditional finite difference method (FDM). Transversal displacements of the deep-sea robot with different methods are shown in Figure 4, where dashdot black, dotted blue and solid lines represent the numerical results by 6 BSWI$_{3}$ elements, 100 FDM elements and MSC.ADAMS simulation, respectively. 


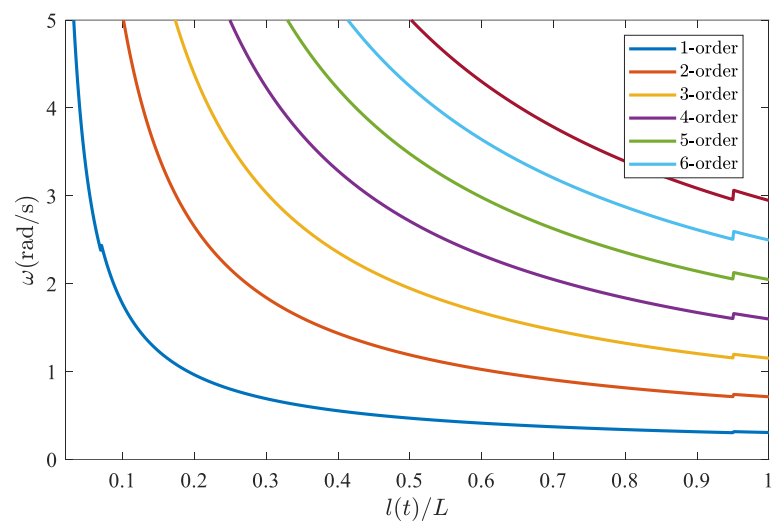

Figure 3. Transversal system frequency.
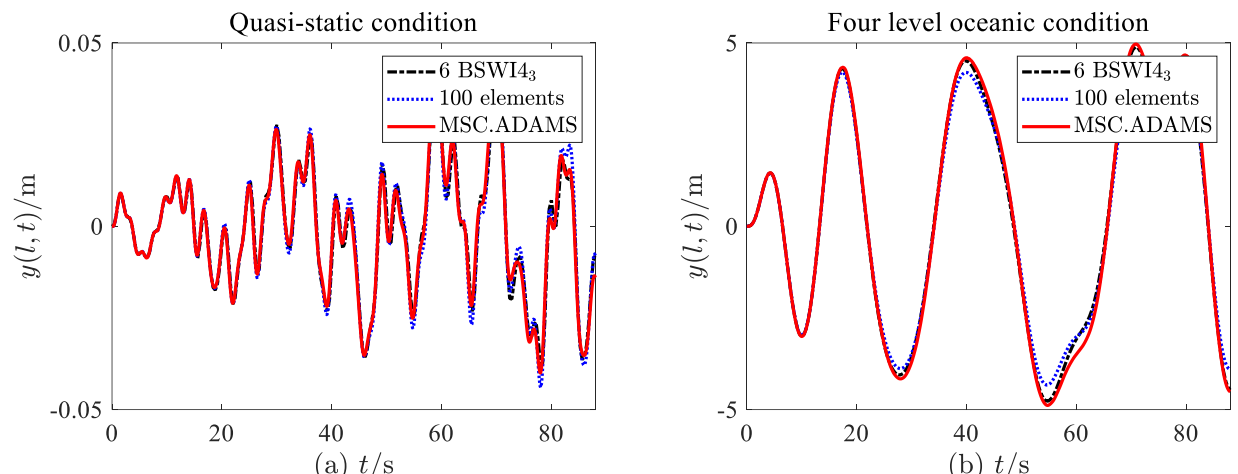

Figure 4. Transverse displacement of the tethered deep-sea robot with boundary disturbance: dashdot black, dotted blue and solid lines represented by 6 BSWI43 elements, 100 elements and MSC.ADAMS, respectively. (a) quasi-static condition; (b) four-level oceanic condition.

When the surface vessel is in quasi-static oceanic condition, the umbilical cable vibrates around the equilibrium position with a small transversal displacement of only $0.045 \mathrm{~m}$. In a four-level oceanic condition, it presents a significant vibration with a low disturbance frequency. The flexible umbilical cable suffers from boundary disturbance of the surface vessel, whose vibrational behavior depends on natural cable frequency (and transversal wavelength) of the sea waves reflecting back and forth from the ends. When the disturbance frequency approaches the first-order natural frequency with the increasing umbilical cable length, the transverse vibration grows larger and larger. The accuracy of the FDM is directly related to the time step $\Delta t$ and mesh size $n$ of the umbilical cable, however, the computational effort will increase with the more minor time step and more number elements. Besides, traditional FDM will reduce computational accuracy because of the errors brought from processing the boundary by using some ghost points [32]. Simulation results for transverse displacement of the deep-sea robot demonstrated the high computational accuracy in solving the nonhomogeneous PDEs compared to the traditional FDM.

\section{Different Cases of Transverse Vibrations for the Tethered Deep-Sea Robot System}

Since the scaling function works as the basis function for the BSWI finite element, the high accuracy of explicit element expression reflects the superiority of BSWI finite element in providing a variety of optional basis functions for structural analysis, where the scaling functions with different orders and scales can be selected to construct basis function to meet the accuracy requirements. In addition, the accuracy of strain expression can be improved by increasing the number of elements and increasing the order of BSWI, and transforming matrix of BSWI finite element method is used to deal with PDEs with nonhomogeneous boundary conditions. 
The spatial shape and displacement of the tethered deep-sea robot explicitly depend on comprehensive effects from internal and external environments, such as the transversal motion of the supporting mother and unexpected ocean current, etc. The umbilical cable is no longer a vertical line in shape, but it presents a space curve. Among current literatures, most scholars focus on the static deformation of the umbilical cable, without considering the comprehensive effects of the two disturbances. The steady-state analysis of the system is usually time-independent, and the dynamic characteristics of the underwater umbilical cable changes over time are rarely analyzed. Spatial shape and displacement of the umbilical cable at different cases are compared in this section: (1) Quasi-static; (2) Current disturbance (3) Boundary disturbance; and (4) Mixed disturbance including current and boundary disturbance.

The 3D spatial shape and displacement of the deep-sea robot are shown as Figure 5, where hydrodynamic parameters are given as the Section 3. In 3D spatial shape of the umbilical cable, the coordinate $x=0$ indicates the surface vessel, where its disturbance displacement is simplified to a trigonometric function, and the coordinate $x=1$ indicates the transversal displacement of the tethered deep-sea robot with normalized position. The solid lines stand for spatial shape of the umbilical cable with different times in b in Figure 5.
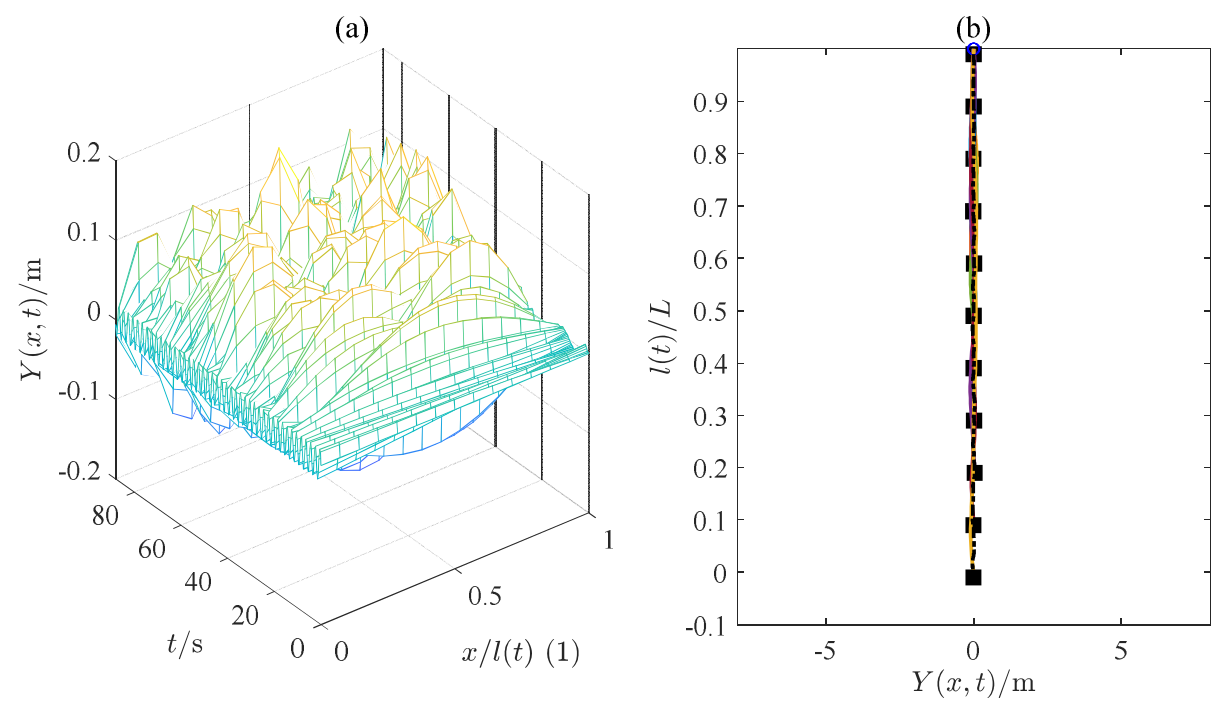

(A). Quasi-static

(a)

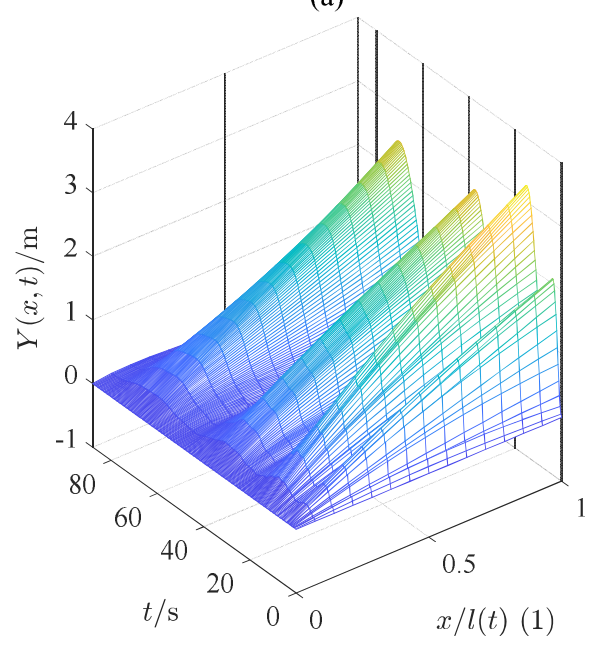

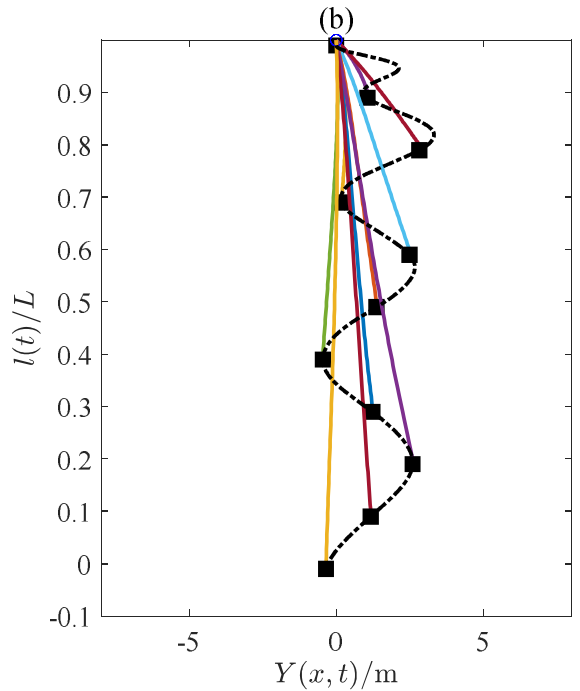

(B). Current disturbance

Figure 5. Cont. 
(a)

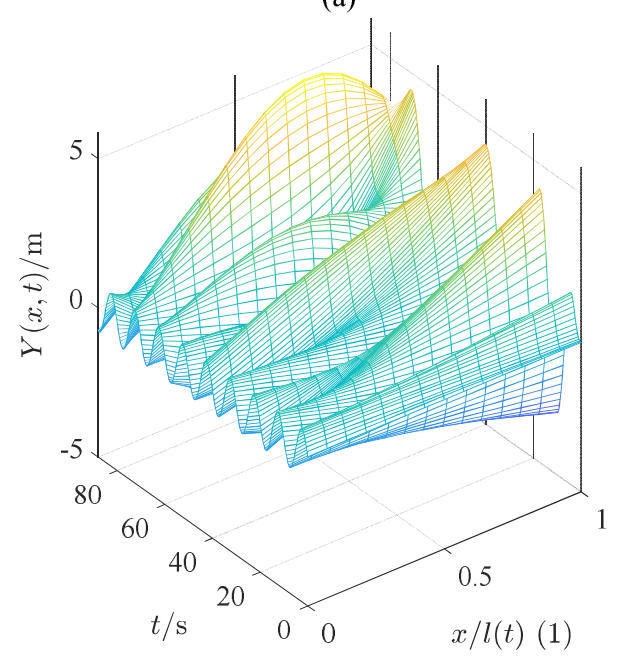

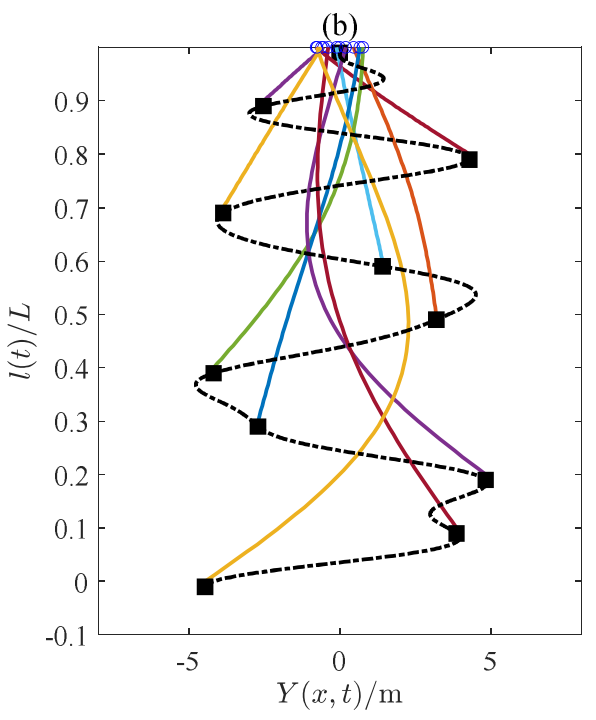

(C). Boundary disturbance

(a)

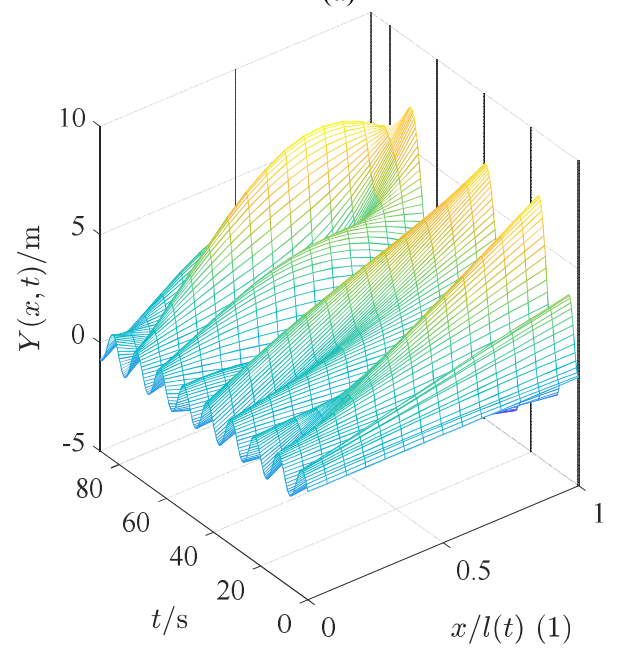

(b)

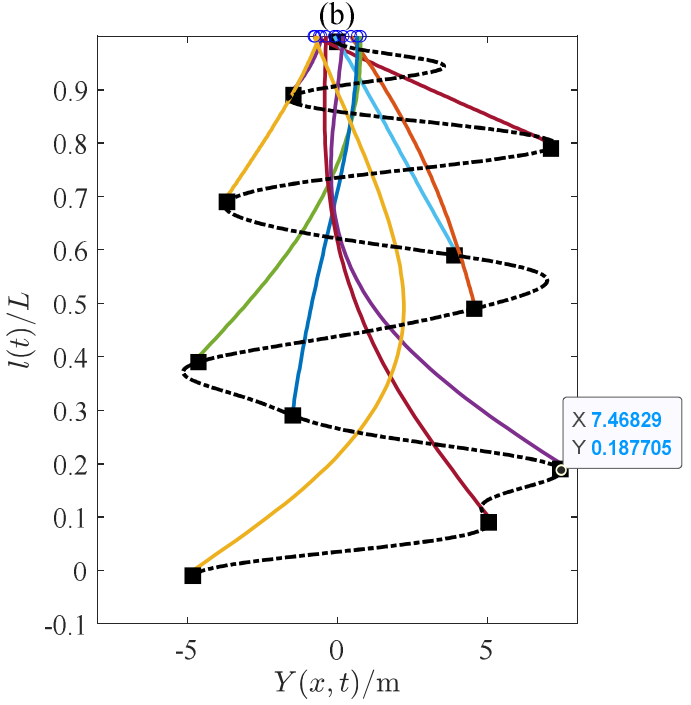

(D). Mixed disturbance

Figure 5. Spatial shape and displacement of the deep-sea robot: (a) 3D Spatial shape of the umbilical cable; (b) Small black box, solid line and circle line stands for the deep-sea robot, the umbilical cable and the surface vessel, respectively.

In the quasi-static case, the spatial displacement of the umbilical cable is almost a vertical line. The transverse displacement of the tethered deep-sea robot, indicated by a black dash dot line, increases with diving depth in other cases. Under the action of ocean current disturbance, the umbilical cable shifts to the direction of distributed load, but returns to the initial equilibrium point with its own gravity, where the maximum transverse displacement is $2.5 \mathrm{~m}$. Compared with the motion amplitude of the surface vessel, the maximum transverse displacement of the umbilical cable is equal to $7.48 \mathrm{~m}$ of the mixed disturbance case, which is amplified more than nine times in the transverse direction.

The dynamic tension of the umbilical cable is related to the position deviation of the transverse displacement. From the above calculation results, we can get the dynamic tension of the umbilical cable when the transverse displacement of each point on the rope is known. The umbilical cable on the boundary of the surface vessel and the tethered deep-sea robot force are more concerned since is generally large. The action of the force is mutual, the reacting force from the umbilical cable is shown in Figure 6. 


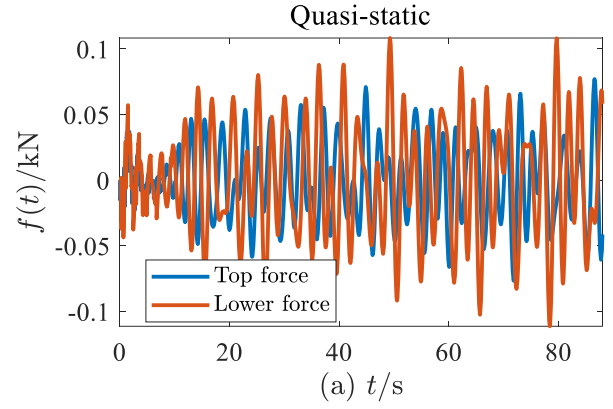

Current disturbance

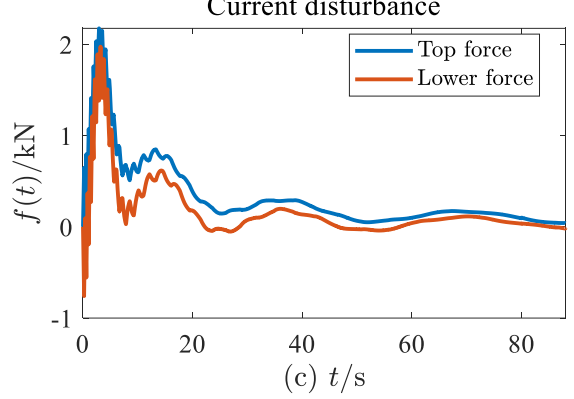

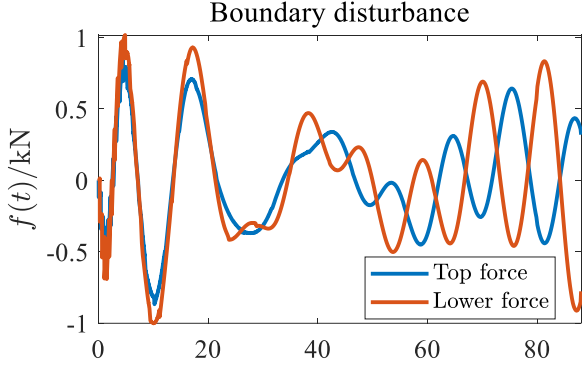

(b) $t / \mathrm{s}$

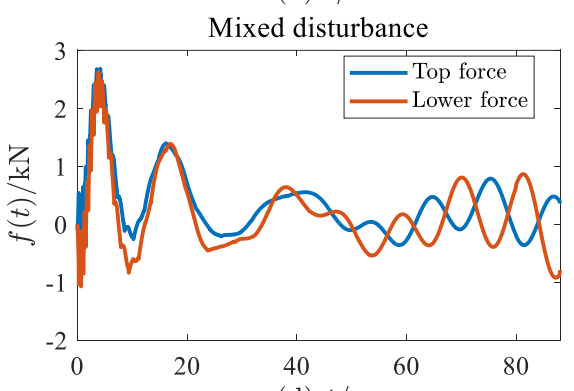

(d) $t / \mathrm{s}$

Figure 6. Reacting force from the umbilical cable: (a) Quasi-static; (b) Boundary disturbance; (c) Current disturbance and (d) Mixed disturbance.

Under the quasi-static condition, the tension fluctuation is small, and the fluctuation range of the combined tension is within $100 \mathrm{~N}$ shown in Figure 6a. Different cases of boundary and ocean current disturbances will cause reversal loading of the umbilical cable with more considerable value, and the maximum reacting force of the mixed disturbance is more extensive than that only boundary disturbance. Its service life is shortened due to unexpected vibration and alternating stress. It can be seen from the graph that the boundary disturbance has the most significant influence on the dynamic tension of the umbilical cable, followed by the ocean current disturbance.

\section{Conclusions}

In this paper, a new numerical method with BSWI finite element is presented to approximate the transverse vibration of the deep-sea robot with time-varying length. A dynamic model of the system is a one-dimensional time-dependent of the hyperbolic PDEs problem derived by using Hamilton principle. Some specific piecewise functions are selected as BSWI scaling functions, thereby the transverse vibration problem involved in the BSWI finite element mapped from the wavelet space to the physical space. By using BSWI finite element and the spatial-temporal discretization, the dynamic model of the system can be obtained as a set of the differential equation. For the convenience of calculation, Gauss-Legendre numerical method is proposed to obtain multiple integrations of the dynamic model. The calculation results followed by RK45 are more consistent with that of ADAMS simulation. Compared with traditional methods, the proposed method approximates to the actual solution using more less elements, and the results of the BSWI finite element method is more accurate than that of the traditional FDM. Through the natural frequency analysis of the system, the transverse frequency of the system becomes lower and lower as the length of the umbilical increases, when disturbance frequency of the surface vessel and ocean current is closed to the first-order natural of the system, the transverse vibration of the deep-sea robot is more and more prominent. This paper mainly discusses the factors affecting maximum transverse displacement of the umbilical cable, such as boundary disturbance and ocean current disturbance. 


\begin{abstract}
Author Contributions: N.W.: Data curation, Writing—original draft, Software, Validation, Visualization, Conceptualization, Funding acquisition, Project administration. X.X.: Investigation, Validation, Methodology, Visualization, Formal analysis, Writing—review \& editing. All authors have read and agreed to the published version of the manuscript.
\end{abstract}

Funding: This research was funded by the National Natural Science Foundation of China (Grant No.52005373); Zhejiang Provincial Natural Science Foundation of China (Grant No. LQ21E050002); Wenzhou Municipal Science and Technology Bureau, China (Grant No. G2020014).

Institutional Review Board Statement: Not applicable.

Informed Consent Statement: Not applicable.

Data Availability Statement: Not applicable.

Conflicts of Interest: The authors declare that they have no known competing financial interests or personal relationships that could have appeared to influence the work reported in this paper.

\title{
References
}

1. Driscoll, F.R.; Lueck, R.G.; Nahon, M. Development and validation of a lumped-mass dynamics model of a deep-sea ROV system. Appl. Ocean Res. 2000, 22, 169-182. [CrossRef]

2. Zen, G.; Müftü, S. Stability of an axially accelerating string subjected to frictional guiding forces. J. Sound Vib. 2006, 289, 551-576. [CrossRef]

3. Koivurova, H. The numerical study of the nonlinear dynamics of a light, axially moving string. J. Sound Vib. 2009, 320, 373-385. [CrossRef]

4. Tavasoli, A. Active disturbance rejection boundary control of Timoshenko beam with tip mass. ISA Trans. 2018, 80, 221-231. [CrossRef] [PubMed]

5. Liu, X.; Zhou, Y.; Wang, X.; Wang, J. A wavelet method for solving a class of nonlinear boundary value problems. Commun. Nonlinear Sci. 2013, 18, 1939-1948. [CrossRef]

6. Priyadarshi, G.; Rathish Kumar, B.V. Wavelet Galerkin method for fourth order linear and nonlinear differential equations. Appl. Math. Comput. 2018, 327, 8-21. [CrossRef]

7. Kang, H.; Guo, T.; Zhu, W. Multimodal interaction analysis of a cable-stayed bridge with consideration of spatial motion of cables. Nonlinear Dynam. 2019, 99, 123-147. [CrossRef]

8. Lee, S.-Y.; Mote, C.D. Vibration Control of an Axially Moving String by Boundary Control. Journal of Dynamic Systems. Meas. Control 1996, 118, 66-74. [CrossRef]

9. Wickert, J.A.; Mote, C.D. Classical Vibration Analysis of Axially Moving Continua. J. Appl. Mech. 1990, 57, 738-744. [CrossRef]

10. Tan, C.A.; Mote, C.D. Analysis of a Hydrodynamic Bearing Under Transverse Vibration of an Axially Moving Band. J. Tribol. 1990, 112, 514-523. [CrossRef]

11. Wang, C.Y.; Wang, C.M. Exact solutions for vibration of a vertical heavy string with a tip mass. IES J. Part A Civil Struct. Eng. 2010, 3, 278-281. [CrossRef]

12. Reddy, J.N.; Berry, J. Nonlinear theories of axisymmetric bending of functionally graded circular plates with modified couple stress. Compos. Struct. 2012, 94, 3664-3668. [CrossRef]

13. Reddy, J.N. Nonlocal theories for bending, buckling and vibration of beams. Int. J. Eng. Sci. 2007, 45, 288-307. [CrossRef]

14. Şimşek, M.; Reddy, J.N. Bending and vibration of functionally graded microbeams using a new higher order beam theory and the modified couple stress theory. Int. J. Eng. Sci. 2013, 64, 37-53. [CrossRef]

15. Xiang, J.; Chen, X.; Mo, Q.; He, Z. Identification of crack in a rotor system based on wavelet finite element method. Finite Elem. Anal. Des. 2007, 43, 1068-1081. [CrossRef]

16. Xiang, J.; Zhong, Y.; Chen, X.; He, Z. Crack detection in a shaft by combination of wavelet-based elements and genetic algorithm. Int. J. Solids Struct. 2008, 45, 4782-4795. [CrossRef]

17. Wang, Z.; Sun, G. Parameters influence on maneuvered towed cable system dynamics. Appl. Ocean. Res. 2015, 49, 27-41. [CrossRef]

18. Quan, W.; Liu, Y.; Zhang, Z.; Li, X.; Liu, C. Scale model test of a semi-active heave compensation system for deep-sea tethered ROVs. Ocean Eng. 2016, 126, 353-363. [CrossRef]

19. Meirovitch, L. Analytical Methods in Vibration; Macmillan Publishing Co., Inc.: New York, NY, USA, 1967.

20. Wei, Q.; Xiang, J. B-spline wavelet boundary element method for three-dimensional problems. Acta Mech. 2021, $232,3233-3257$. [CrossRef]

21. Xiang, J.W.; Chen, X.F.; He, Z.J.; Dong, H.B. The construction of 1D wavelet finite elements for structural analysis. Comput. Mech. 2006, 40, 325-339. [CrossRef]

22. Wu, J.; Chen, D. Trajectory Following of a Tethered Underwater Robot with Multiple Control Techniques. J. Offshore Mech. Arct. 2019, 141, 1-9. [CrossRef] 
23. Zhong, Y.; Xiang, J. Construction of wavelet-based elements for static and stability analysis of elastic problems. Acta Mech. Solida Sin. 2011, 24, 355-364. [CrossRef]

24. Zhong, Y.; Xiang, J.; Chen, X.; Jiang, Y.; Pang, J. Multiple Signal Classification-Based Impact Localization in Composite Structures Using Optimized Ensemble Empirical Mode Decomposition. Appl. Sci. 2018, 8, 1447. [CrossRef]

25. Chui, C.K.; Quak, E. Wavelets on a Bounded Interval. Numerical Methods in Approximation Theory; Birkhauser Verlag: Basel, Switzerland, 1992; Volume 9, pp. 53-75.

26. Plonka, G.; Selig, K.; Tasche, M. On the construction of wavelets on a bounded interval. Adv. Comput. Math. 1995, 4, 357-388. [CrossRef]

27. Sarpkaya, T. Wave Impact Loads on Cylinders. Soc. Petroleum Eng. J. 1979, 19, 29-36. [CrossRef]

28. Reddy, J.N. Principles of Continuum Mechanics: A Study of Conservation Principles with Applications; Cambridge University Press: New York, USA, 2010.

29. Kuo, Y.L.; Cleghorn, W.L.; Behdinan, K. Relative-Error-Based Finite Element Analysis of Axially Moving Beams. Trans. Can. Soc. Mech. Eng. 2006, 30, 345-358. [CrossRef]

30. Stylianou, M.; Tabarrok, B. Finite Element Analysis of An Axially Moving Beam, Part II: Stability Analysis. J. Sound Vib. 1994, 178, 455-481. [CrossRef]

31. Stylianou, M.; Tabarrok, B. Finite Element Analysis of An Axially Moving Beam, Part I: Time Integration. J. Sound Vib. 1994, 178, 433-453. [CrossRef]

32. Coco, A.; Russo, G. Finite-difference ghost-point multigrid methods on Cartesian grids for elliptic problems in arbitrary domains. J. Comput. Phys. 2013, 241, 464-501. [CrossRef] 